\title{
A New Error Analysis for a Cubic Spline Approximate Solution of a Class of Volterra Integro-Differential Equations
}

\author{
By Joseph A. Guzek and Gene A. Kemper
}

\begin{abstract}
In this paper a third-order numerical method is considered which utilizes a twice continuously differentiable third degree spline to approximate the solution of

$$
\begin{aligned}
& \dot{x}(t)=F\left(t, x(t), \int_{a}^{t} K(t, u, x(u)) d u\right), \\
& x(a)=x_{0},
\end{aligned}
$$

at discrete points in the interval $[a, b]$. The error analysis uses a technique usually associated with linear multistep methods.
\end{abstract}

I. Introduction. In this paper, consideration is directed to the Volterra integrodifferential equation

$$
\dot{x}(t)=F\left(t, x(t), \int_{a}^{t} K(t, u, x(u)) d u\right), \quad a \leqq t \leqq b,
$$

with the initial condition $x(a)=x_{0}$. A third-order numerical method is considered which utilizes a twice continuously differentiable third degree spline to approximate the solution $x$ at discrete points in the interval $[a, b]$.

Other authors, e.g. Hung [5], have applied cubic splines to obtain an approximate solution of a scalar Volterra integro-differential equation. This paper considers the method as applied to vector equations. More important, however, is the error analysis presented herein. This analysis uses a lemma usually associated with linear multistep methods. The utilization of this lemma allows the cubic spline method to be applied to a larger class of equations than considered by Hung with, however, a corresponding reduction in the order of the errors. In particular, Hung requires the solution of (1) be of class $C^{6}[a, b]$ while the analysis presented here requires only $C^{4}[a, b]$. Accordingly, Hung achieves a discretization error $O\left(h^{4}\right)$ while this analysis achieves $O\left(h^{3}\right)$.

II. Notation and Assumptions. Let $R(F)$ and $R(K)$ be the regions defined by

$$
R(F)=\left\{(t, x, y): a \leqq t \leqq b ; x, y \in E^{n}\right\}
$$

and

$$
R(K)=\left\{(t, u, y): a \leqq u \leqq t \leqq b ; y \in E^{n}\right\},
$$

Received April 4, 1972.

AMS (MOS) subject classifications (1970). Primary 65R05; Secondary 45D05.

Key words and phrases. Volterra integro-differential equations, spline approximation.

Copyright (C) 1973, American Mathematical Society 
where $E^{n}$ is real Euclidean $n$-space. Moreover, let the $n$th order matrices $F^{(2)}, F^{(3)}$, and $K^{(3)}$ be such that the respective elements are given by

$$
F_{i, j}^{(2)}=\frac{\partial F_{i}}{\partial x_{i}}, \quad F_{i, j}^{(3)}=\frac{\partial F_{i}}{\partial y_{i}}, \quad \text { and } \quad K_{i, i}^{(3)}=\frac{\partial K_{i}}{\partial y_{i}}
$$

Then, the following assumptions are made:

(a) Equation (1) has a unique solution.

(b) $F$ and $K$ are continuous mappings of $R(F)$ and $R(K)$ to $E^{n}$, respectively.

(c) The matrix elements (2) are continuous and bounded.

Assumption (c) has two important implications. First, there exist constants $\bar{F}^{(2)}, \bar{F}^{(3)}$ and $\bar{K}^{(3)}$ such that $\left\|F^{(2)}\right\| \leqq \bar{F}^{(2)},\left\|F^{(3)}\right\| \leqq \bar{F}^{(3)}$ and $\left\|K^{(3)}\right\| \leqq \bar{K}^{(3)}$, where $\|\cdot\|$ will be used interchangeably to denote compatible matrix and vector norms. Secondly, Buck [1, p. 268], for $(t, x, y),(t, \bar{x}, y) \in R(F)$ there exist $p_{i} \in E^{n}$, $i=1, \cdots, n$, such that

$$
F(t, x, y)-F(t, \bar{x}, y)=F^{(2)}(x-\bar{x})
$$

where $F^{(2)}=\left(F_{i, j}^{(2)}\left(t, p_{i}, y\right)\right)$. Similarly, for $(t, x, y),(t, x, \bar{y}) \in R(F)$ and for $(t, u, v)$, $(t, u, \bar{v}) \in R(K)$, there are $q_{i}, r_{i} \in E^{n}, i=1, \cdots, n$, such that

$$
F(t, x, y)-F(t, x, \bar{y})=F^{(3)}(y-\bar{y})
$$

and

$$
K(t, u, v)-K(t, u, \bar{c})=K^{(3)}(v-\bar{c})
$$

where $F^{(3)}=\left(F_{i, j}^{(3)}\left(t, x, q_{i}\right)\right)$ and $K^{(3)}=\left(K_{i, j}^{(3)}\left(t, u, r_{i}\right)\right)$.

III. The Method. Let $[a, b]$ be divided into $N$ equal subintervals of length $h=(b-a) / N$ with endpoints $t_{0}, t_{1}, \cdots, t_{v}$, called nodes. Let $x_{k}, \dot{x}_{k}$ and $\ddot{x}_{k}$ denote approximations for $x\left(t_{k}\right), \dot{x}\left(t_{k}\right)$ and $\ddot{x}\left(t_{k}\right)$, respectively. The $n$-dimensional cubic spline $S$ on $[a, b]$ is defined as follows: For $t \in\left[t_{k}, t_{k+1}\right], S$ is denoted by $S_{k}$ and is defined by

$$
S_{k}(t)=x_{k}+\left(t-t_{k}\right) \dot{x}_{k}+\frac{\left(t-t_{k}\right)^{2}}{2} \ddot{x}_{k}+\frac{\left(t-\frac{t_{k}}{}\right)^{3}}{3 h^{2}}\left(\dot{x}_{k+1}-\dot{x}_{k}-h \ddot{x}_{k}\right) .
$$

Note that $S_{k}\left(t_{k}\right)=x_{k}, \dot{S}_{k}\left(t_{k}\right)=\dot{x}_{k}, \ddot{S}_{k}\left(t_{k}\right)=\ddot{x}_{k}$ and $\dot{S}_{k}\left(t_{k+1}\right)=\dot{x}_{k+1}$.

The approximate solution to (1) is obtained by replacing the integral by a numerical quadrature formula and requiring that the resulting equation be satisfied at the nodes. Thus, if the cubic spline $S$ replaces $x$ in this equation, (1) is replaced by

$$
\dot{S}_{k}\left(t_{k+1}\right)=F\left(t_{k+1}, S_{k}\left(t_{k+1}\right), h \sum_{i=0}^{k+1} w_{i} K\left(t_{k+1}, t_{i}, S_{i-1}\left(t_{i}\right)\right)\right)
$$

where the weights $w_{i}$ are bounded and depend on the numerical quadrature formula used and where $S_{-1}\left(t_{0}\right) \equiv x_{0}$. Then, using $x_{k}=S_{k-1}\left(t_{k}\right), \dot{x}_{k}=\dot{S}_{k-1}\left(t_{k}\right), \ddot{x}_{k}=\ddot{S}_{k-1}\left(t_{k}\right)$ and $\dot{x}_{k+1}=\dot{S}_{k}\left(t_{k+1}\right)$, (4) becomes

$$
\dot{x}_{k+1}=H\left(\dot{x}_{k+1}\right)
$$


where

$$
H\left(\dot{x}_{k+1}\right)=F\left(t_{k+1}, x_{k}+h \dot{x}_{k}+\frac{h^{2}}{2} \ddot{x}_{k}+\frac{h}{3}\left(\dot{x}_{k+1}-\dot{x}_{k}-h \ddot{x}_{k}\right), q_{k+1}\right)
$$

with

$$
\begin{aligned}
q_{k+1}= & h \sum_{i=0}^{k} w_{i} K\left(t_{k+1}, t_{i}, x_{i}\right) \\
& +h w_{k+1} K\left(t_{k+1}, t_{k+1}, x_{k}+h \dot{x}_{k}+\frac{h^{2}}{2} \ddot{x}_{k}+\frac{h}{3}\left(\dot{x}_{k+1}-\dot{x}_{k}-h \ddot{x}_{k}\right)\right) .
\end{aligned}
$$

All quantities in (5) are known except $\dot{x}_{k+1}$. Since $\dot{x}_{k+1}$ determines $S_{k}$, the values $x_{k+1}=S_{k}\left(t_{k+1}\right)$ and $\ddot{x}_{k+1}=\ddot{S}_{k}\left(t_{k+1}\right)$ follow. (Although (5) is used to determine $x_{k}$, it is convenient to use (4) in the error analysis to follow.)

It follows, in the usual straightforward manner, from assumption (c) that, for $x$, $\bar{x} \in E^{n}$

$$
\|H(x)-H(\bar{x})\| \leqq \frac{h \bar{F}^{(2)}+h^{2}\left|w_{k+1}\right| \bar{F}^{(3)} \bar{K}^{(3)}}{3}\|x-\bar{x}\|
$$

Thus, for $h$ sufficiently small the mapping given by (5) is a contraction. This proves the following theorem.

THEOREM 1. For $H$ as defined by (5) and with assumption (c) satisfied, it follows that, for sufficiently small $h, H$ is a contraction mapping.

Thus, (5) can be used iteratively to determine $x_{i}, i=r, \cdots, N$, where $r$ depends on the starting method used.

IV. Error Analysis. Let $E(t)=x(t)-S(t)$. Then, from (1) and (4), there follows

$$
\begin{aligned}
\dot{E}\left(t_{k}\right)= & F\left(t_{k}, x\left(t_{k}\right), \int_{t_{0}}^{t_{k}} K\left(t_{k}, u, x(u)\right) d u\right)-F\left(t_{k}, S_{k-1}\left(t_{k}\right), \int_{t_{0}}^{t_{k}} K\left(t_{k}, u, x(u)\right) d u\right) \\
& +F\left(t_{k}, S_{k-1}\left(t_{k}\right), \int_{t_{0}}^{t_{k}} K\left(t_{k}, u, x(u)\right) d u\right) \\
& -F\left(t_{k}, S_{k-1}\left(t_{k}\right), h \sum_{i=0}^{k} w_{i} K\left(t_{k}, t_{i}, x\left(t_{i}\right)\right)\right) \\
& +F\left(t_{k}, S_{k-1}\left(t_{k}\right), h \sum_{i=0}^{k} w_{i} K\left(t_{k}, t_{i}, x\left(t_{i}\right)\right)\right) \\
& -F\left(t_{k}, S_{k-1}\left(t_{k}\right), h \sum_{i=0}^{k} w_{i} K\left(t_{k}, t_{i}, S_{i-1}\left(t_{i}\right)\right)\right) .
\end{aligned}
$$

Thus, in view of assumption (c),

$$
\dot{E}\left(t_{k}\right)=F_{(k)}^{(2)} E\left(t_{k}\right)+\widetilde{O}\left(h^{p}\right)+h F_{(k)}^{(3)} \sum_{i=0}^{k} w_{i} K_{(k, i)}^{(3)} E\left(t_{i}\right)
$$


where $F_{(k)}^{(2)}$ and $F_{(k)}^{(3)}$ indicate the matrices $F^{(2)}$ and $F^{(3)}$ depend on the index $k$ and $K_{(k, i)}^{(3)}$ indicates the matrix depends on the indices $k$ and $i$. Furthermore, the numerical quadrature formula is assumed to be such that

$$
\int_{t_{0}}^{t_{k}} K\left(t_{k}, u, x(u)\right) d u-h \sum_{i=0}^{k} w_{i} K\left(t_{k}, t_{i}, x\left(t_{i}\right)\right)=\tilde{O}\left(h^{p}\right)
$$

where $\widetilde{O}\left(h^{\nu}\right)$ is a vector with components all $O\left(h^{p}\right)$.

The error analysis development is to obtain an equation involving $E$ and $\dot{E}$ at the nodes. Then, (6) is used to provide an equation in $E$ only. The error information at the nodes is then used to obtain error bounds at nonnodal points.

To proceed with the error analysis at the nodes, it is assumed the solution $x \in$ $C^{(4)}[a, b]$. Then, for $t \in\left[t_{k}, t_{k+1}\right]$,

$$
\begin{aligned}
& E(t)=E\left(t_{k}\right)+\left(t-t_{k}\right) \dot{E}\left(t_{k}\right)+\frac{\left(t-\frac{t_{k}}{2}\right)^{2}}{2} \ddot{E}\left(t_{k}\right)+\frac{\left(t-t_{k}\right)^{3}}{6} \dddot{E}\left(t_{k}\right)+\tilde{O}\left(h^{4}\right), \\
& \dot{E}(t)=\dot{E}\left(t_{k}\right)+\left(t-t_{k}\right) \ddot{E}\left(t_{k}\right)+\frac{\left(t-\frac{t_{k}}{2}\right)^{2}}{2} \dddot{E}\left(t_{k}\right)+\widetilde{O}\left(h^{3}\right),
\end{aligned}
$$

and

$$
\ddot{E}(t)=\ddot{E}\left(t_{k}\right)+\left(t-t_{k}\right) \dddot{E}\left(t_{k}\right)+\widetilde{O}\left(h^{2}\right) .
$$

(Since $S^{(4)}=0$, the error terms involve only the solution $x$ and not the spline $S$.)

Evaluation of (7) and (8) at $t_{k+1}$ and elimination of $\dddot{E}\left(t_{k}\right)$ provides

$$
E\left(t_{k+1}\right)=E\left(t_{k}\right)+\frac{2 h}{3} \dot{E}\left(t_{k}\right)+\frac{h}{3} \dot{E}\left(t_{k+1}\right)+\frac{h^{2}}{6} \ddot{E}\left(t_{k}\right)+\tilde{O}\left(h^{4}\right)
$$

while elimination of $\ddot{E}\left(t_{k}\right)$ provides

$$
\frac{h^{3}}{12} \dddot{E}\left(t_{k}\right)=E\left(t_{k}\right)-E\left(t_{k+1}\right)+\frac{h}{2} \dot{E}\left(t_{k}\right)+\frac{h}{2} \dot{E}\left(t_{k+1}\right)+\tilde{O}\left(h^{4}\right) .
$$

Evaluation of (9) at $t_{k+1}$ and substitution of $\dddot{E}\left(t_{k}\right)$ from (11) yields

$$
\ddot{E}\left(t_{k+1}\right)=\ddot{E}\left(t_{k}\right)+\frac{12}{h^{2}} E\left(t_{k}\right)-\frac{12}{h^{2}} E\left(t_{k-1}\right)+\frac{6}{h} \dot{E}\left(t_{k}\right)+\frac{6}{h} \dot{E}\left(t_{k+1}\right)+\widetilde{O}\left(h^{2}\right) .
$$

Reduction of subscripts by one in (12) and substitution of the resulting expression for $\ddot{E}\left(t_{k-1}\right)$ into the equation which results from the reduction of the subscripts by one in (10) yields

$$
\frac{h^{2}}{6} \ddot{E}\left(t_{k}\right)=-E\left(t_{k}\right)+E\left(t_{k-1}\right)+\frac{2 h}{3} \dot{E}\left(t_{k}\right)+\frac{h}{3} \dot{E}\left(t_{k-1}\right)+\widetilde{O}\left(h^{4}\right),
$$

which when substituted in (10) provides

$$
E\left(t_{k+1}\right)-E\left(t_{k-1}\right)+\frac{h}{3}\left[\dot{E}\left(t_{k-1}\right)+4 \dot{E}\left(t_{k}\right)+\dot{E}\left(t_{k+1}\right)\right]+\widetilde{O}\left(h^{4}\right) .
$$

Finally, from (6) and (13), there follows 


$$
\begin{aligned}
& E\left(t_{k+1}\right)-E\left(t_{k-1}\right)=\frac{h}{3}\left[F_{(k-1)}^{(2)} E\left(t_{k-1}\right)+4 F_{(k)}^{(2)} E\left(t_{k}\right)+F_{(k+1)}^{(2)} E\left(t_{k+1}\right)\right] \\
& +\frac{h^{2}}{3}\left[F_{(k-1)}^{(3)} \sum_{i=0}^{k-1} w_{i} K_{(k-1, i)}^{(3)} E\left(t_{i}\right)+4 F_{(k)}^{(3)} \sum_{i=0}^{k} w_{i} K_{(k, i)}^{(3)} E\left(t_{i}\right)\right. \\
& \left.+F_{(k+1)}^{(3)} \sum_{i=0}^{k+1} w_{i} K_{(k+1, i)}^{(3)} E\left(t_{i}\right)\right] \\
& +\widetilde{O}\left(h^{\min (p+1,4)}\right) .
\end{aligned}
$$

In order to bound the discretization errors at the nodes, the following lemma is used, the proof of which is similar to that for Lemma 5.6 (Henrici, [3, p. 243]) and Linz's [7] lemma.

LEMMA. Let $z_{m} \in E^{n}, m \geqq r$, be the solution of

$$
\begin{aligned}
\rho_{k} z_{m+k}+\cdots+\rho_{0} z_{m} & =h\left(\beta_{k, m} z_{m+k}+\beta_{k-1, m+k-1} z_{m+k-1}+\cdots+\beta_{0, m} z_{m}\right) \\
+ & h^{2}\left(\sum_{i=0}^{m+k} \mu_{m+k, i} z_{i}+\sum_{i=0}^{m+k-1} \mu_{m+k-1, i} z_{i}+\cdots+\sum_{i=0}^{m} \mu_{m, i} z_{i}\right)+\lambda_{m}
\end{aligned}
$$

where all $\beta_{i, j}$ and $\mu_{i, j}$ are nth order matrices and the $\rho_{i}$ are scalars. Assume the polynomial $\rho_{k} \xi^{k}+\rho_{k-1} \xi^{k-1}+\cdots+\rho_{0}$ satisfies the Dahlquist stability condition (Henrici $[3, p .218])$. Thus, if

$$
1 /\left(\rho_{k}+\rho_{k-1} \xi+\cdots+\rho_{0} \xi^{k}\right) \equiv \gamma_{0}+\gamma_{1} \xi+\cdots,
$$

where $\rho_{k} \neq 0$, then $\Gamma \equiv \sup _{i}\left|\gamma_{i}\right|<\infty$ (Henrici [3, p. 242]). Furthermore, assume $\left\|z_{i}\right\| \leqq Z, i=0,1, \cdots, k+r-1$, and for all $i, j,\left\|\beta_{i, j}\right\| \leqq \beta,\left\|\mu_{i, j}\right\| \leqq \mu,\left\|\lambda_{i}\right\| \leqq \lambda$. Then, for sufficiently small $h$,

$$
\left\|z_{n}\right\| \leqq K^{*} e^{n h L^{*}}, \quad n=0,1, \cdots, N
$$

where

$$
\begin{aligned}
K^{*} & =\left[k \Gamma A Z+h b \mu^{*} \Gamma r Z+N \lambda \Gamma\right] /\left[1-h \Gamma\left(\beta+b \mu^{*}\right)\right], \\
L^{*} & =\left[\beta^{*} \Gamma+b \mu^{*} \Gamma\right] /\left[1-h \Gamma\left(\beta+b \mu^{*}\right)\right], \\
A & =\left|\rho_{0}\right|+\cdots+\left|\rho_{n}\right| \\
\beta^{*} & =\beta(k+1) \text { and } \mu^{*}=\mu(k+1) .
\end{aligned}
$$

Application of the lemma to (14) yields

$$
\left\|E\left(t_{k}\right)\right\| \leqq K^{*} e^{L^{*} t_{k}}
$$

with

$$
K^{*}=\left[4 Z+4 h b w \bar{K}^{(3)} \bar{F}^{(3)} r Z+b O\left(h^{\min (3, p)}\right)\right] /\left[1-h\left(\frac{4}{3} \bar{F}^{(2)}+4 b w \bar{F}^{(3)} \bar{K}^{(3)}\right)\right]
$$

and

$$
L^{*}=\left[4 \bar{F}^{(2)}+4 b w \bar{F}^{(3)} \bar{K}^{(3)}\right] /\left[1-h\left(\frac{4}{3} \bar{F}^{(2)}+4 b w \bar{F}^{(3)} \bar{K}^{(3)}\right)\right]
$$

where $\left|w_{i}\right| \leqq w, i=0, \cdots, N . Z$ and $r$ depend on the starting method while $p$ depends on the numerical integration method used. From $K^{*}$ and $L^{*}$, it is readily seen that 
minimally the starting method should be $\widetilde{O}\left(h^{3}\right)$ and $p=3$. For this case $\left\|E\left(t_{k}\right)\right\|=$ $O\left(h^{3}\right)$. There follows from (6) and (10), respectively, that $\left\|\dot{E}\left(t_{k}\right)\right\|=O\left(h^{3}\right)$ and $\left\|\ddot{E}\left(t_{k}\right)\right\|$ $=O(h)$. This proves the following theorem.

THEOREM 2. If assumptions (a), (b) and (c) are satisfied, $x \in C^{(4)}[a, b]$ and the starting method and numerical integration method are both $\widetilde{O}\left(h^{3}\right)$, then $\left\|E\left(t_{k}\right)\right\|=$ $O\left(h^{3}\right),\left\|\dot{E}\left(t_{k}\right)\right\|=O\left(h^{3}\right)$ and $\left\|\ddot{E}\left(t_{k}\right)\right\|=O(h)$.

The error analysis at nonnodal points proceeds by setting $t=t_{k+1}$ in (7) and (8), solving the resulting equations for $\ddot{E}\left(t_{k}\right)$ and $\dddot{E}\left(t_{k}\right)$ and substituting these equations back into (7) and (8) to obtain

$$
\begin{aligned}
E(t)= & {\left[1-\frac{3\left(t-t_{k}\right)^{2}}{h^{2}}+\frac{2\left(t-t_{k}\right)^{3}}{h^{3}}\right] E\left(t_{k}\right)+\left[\frac{3\left(t-t_{k}\right)^{2}}{h^{2}}-\frac{2\left(t-t_{k}\right)^{3}}{h^{3}}\right] E\left(t_{k+1}\right) } \\
& +\left[\left(t-t_{k}\right)-\frac{2\left(t-t_{k}\right)^{2}}{h}+\frac{\left(t-t_{k}\right)^{3}}{h^{2}}\right] \dot{E}\left(t_{k}\right) \\
& +\left[\frac{\left(t-t_{k}\right)^{3}}{h^{2}}-\frac{\left(t-t_{k}\right)^{2}}{h}\right] \dot{E}\left(t_{k+1}\right)+\widetilde{O}\left(h^{4}\right)
\end{aligned}
$$

and

$$
\begin{aligned}
\dot{E}(t)= & {\left[\frac{6\left(t-t_{k}\right)^{2}}{h^{3}}-\frac{6\left(t-t_{k}\right)}{h^{2}}\right] E\left(t_{k}\right)+\left[\frac{6\left(t-t_{k}\right)}{h^{2}}-\frac{6\left(t-t_{k}\right)^{2}}{h^{3}}\right] E\left(t_{k+1}\right) } \\
& +\left[1-\frac{4\left(t-t_{k}\right)}{h}+\frac{9\left(t-t_{k}\right)^{2}}{h^{2}}\right] \dot{E}\left(t_{k}\right)+\left[\frac{3\left(t-t_{k}\right)^{2}}{h^{2}}-\frac{2\left(t-t_{k}\right)}{h}\right] \dot{E}\left(t_{k+1}\right) \\
& +\tilde{O}\left(h^{3}\right) .
\end{aligned}
$$

Hence, for $t_{k} \leqq t \leqq t_{k+1}$,

$$
\|E(t)\| \leqq\left\|E\left(t_{k}\right)\right\|+\left\|E\left(t_{k+1}\right)\right\|+h\left\|\dot{E}\left(t_{k}\right)\right\|+h\left\|\dot{E}\left(t_{k+1}\right)\right\|+O\left(h^{4}\right)
$$

and

$$
\|\dot{E}(t)\| \leqq 6\left(\frac{1}{h}\left\|E\left(t_{k}\right)\right\|+\frac{1}{h}\left\|E\left(t_{k+1}\right)\right\|+\left\|\dot{E}\left(t_{k}\right)\right\|+\left\|\dot{E}\left(t_{k+1}\right)\right\|\right)+O\left(h^{3}\right) .
$$

Thus, $\|E(t)\|=O\left(h^{3}\right)$ and $\|\dot{E}(t)\|=O\left(h^{2}\right)$. From (9) and (11) there follows $\|\ddot{E}(t)\|=$ $O(h)$. This proves the following theorem.

THEOREM 3. If the conditions of Theorem 2 are satisfied, then, for $t \in[a, b]$, t a nonnodal point, $\|E(t)\|=O\left(h^{3}\right),\|\dot{E}(t)\|=O\left(h^{2}\right)$ and $\|\ddot{E}(t)\|=O(h)$.

V. A Numerical Example. The scalar equation considered here is

$$
\begin{aligned}
& \dot{x}(t)=-\frac{3}{16}(t-1) x(t)+\int_{0}^{t} x(u) d u+\frac{13}{3}(t-1)^{10 / 3}+\frac{3}{16}, \\
& x(0)=-1,
\end{aligned}
$$

which has the solution $x(t)=(t-1)^{13 / 3}$. Gregory's third-order formula,

$$
\int_{t_{0}}^{t_{k}} f \doteq h\left(\frac{5}{12} f_{0}+\frac{13}{12} f_{1}+f_{2}+\cdots+f_{k-2}+\frac{13}{12} f_{k-1}+\frac{5}{12} f_{k}\right),
$$


is used for numerical quadrature. In order to apply Gregory's formula, the values $x_{0}, x_{1}, x_{2}$ and $x_{3}$ are needed. $x_{0}$ is known. To obtain the other starting values, let $G(t, x(t))$ represent the right side of the above equation, i.e., $\dot{x}(t)=G(t, x(t))$, and let $G_{k}=G\left(t_{k}, x\left(t_{k}\right)\right)$. Then

$$
x\left(t_{k+1}\right)=x\left(t_{k}\right)+\int_{t_{k}}^{t_{k+1}} G(t, x(t)) d t
$$

and

$$
x\left(t_{k+2}\right)=x\left(t_{k}\right)+\int_{t_{k}}^{t_{k+2}} G(t, x(t)) d t .
$$

Thus, using Simpson's rule,

$$
x_{k+1}=x_{k}+\frac{h}{6}\left(G_{k}+4 G_{k+1 / 2}+G_{k+1}\right)
$$

and

$$
x_{k+2}=x_{k}+\frac{h}{3}\left(G_{k}+4 G_{k+1}+G_{k+2}\right)
$$

where $t_{k+1 / 2}=t_{k}+h / 2$. The quadratic equation through the points $\left(t_{k}, x_{k}\right),\left(t_{k+1}\right.$, $\left.x_{k+1}\right)$ and $\left(t_{k+2}, x_{k+2}\right)$ evaluated at $t_{k+1 / 2}$ provides

$$
x_{k+1 / 2}=\frac{3}{8} x_{k}+\frac{3}{4} x_{k+1}-\frac{1}{8} x_{k+2}
$$

and, similarly,

$$
G_{k+1 / 2}=\frac{3}{8} G_{k}+\frac{3}{4} G_{k+1}-\frac{1}{8} G_{k+2} .
$$

TABLE OF ERRORS

\begin{tabular}{cccc}
\hline $\begin{array}{c}\text { Step Size } 2^{-P} \\
P\end{array}$ & $|E(1.5)|$ & $|\dot{E}(1.5)|$ & $\begin{array}{c}\max \left|E\left(t_{i}\right)\right|, i=1,2,3 \\
(\text { for starting method })\end{array}$ \\
\hline 2 & $.865964 \times 10^{-3}$ & $.170270 \times 10^{-2}$ & $.636479 \times 10^{-2}$ \\
3 & $.915525 \times 10^{-3}$ & $.101191 \times 10^{-2}$ & $.423563 \times 10^{-3}$ \\
& $\left(.108248 \times 10^{-3}\right)$ & $\left(.212838 \times 10^{-3}\right)$ & \\
4 & $.227987 \times 10^{-3}$ & $.218648 \times 10^{-3}$ & $.274553 \times 10^{-4}$ \\
& $\left(.114481 \times 10^{-3}\right)$ & $\left(.126889 \times 10^{-3}\right)$ & \\
5 & $.380606 \times 10^{-4}$ & $.352258 \times 10^{-4}$ & $.174974 \times 10^{-5}$ \\
& $\left(.284984 \times 10^{-4}\right)$ & $\left(.273310 \times 10^{-4}\right)$ & \\
6 & $.545480 \times 10^{-5}$ & $.498293 \times 10^{-5}$ & $.110465 \times 10^{-6}$ \\
& $\left(.475757 \times 10^{-5}\right)$ & $\left(.440322 \times 10^{-5}\right)$ & \\
7 & $.728986 \times 10^{-6}$ & $.662161 \times 10^{-6}$ & $.693943 \times 10^{-8}$ \\
& $\left(.681850 \times 10^{-6}\right)$ & $\left(.622616 \times 10^{-6}\right)$ & \\
8 & $.941891 \times 10^{-7}$ & $.853280 \times 10^{-7}$ & $.434832 \times 10^{-9}$ \\
& $\left(.911232 \times 10^{-7}\right)$ & $\left(.827701 \times 10^{-7}\right)$ & \\
\hline
\end{tabular}


Equations (15), (16), (17) and (18) are used iteratively to find $x_{1}$ and $x_{2}$, then $x_{3}$ and $x_{4}$. The value $x_{4}$ is not a needed starting value but it is obtained simultaneously with $x_{3}$. The method is used until the difference between two consecutive iterates does not exceed $10^{-12}$. This starting method is $O\left(h^{4}\right)$.

Once the starting values are known, the method of Section III is used with Gregory's formula for the numerical integration. Note the method is applied to $\dot{x}_{i}$, not $x_{i}$. Once $\dot{x}_{i}$ is known, then

$$
x_{i}=S_{i-1}\left(t_{i}\right) \text { and } \quad \ddot{x}_{i}=\ddot{S}_{i-1}\left(t_{i}\right) \text {. }
$$

The above table summarizes the errors corresponding to various step sizes. According to the theory, if the step size is halved the errors in $E$ and $\dot{E}$ should be reduced by approximately one-eighth. The numbers in parenthesis are one-eighth the error for the previous step size, i.e., the error predicted by the theory when the step size is halved.

Department of Mathematics and the Computer Center

University of North Dakota

Grand Forks, North Dakota 58201

1. R. C. Buck, Advanced Calculus, 2nd ed., McGraw-Hill, New York, 1965. MR 42 \#431.

2. J. A. GuzeK \& G. A. Kemper, A Cubic Spline Approximate Solution of a Class of Integro-Differential Equations, Proc. Conf. Numerical Mathematics, University of Manitoba, October 1971.

3. P. HenRICI, Discrete Variable Methods in Ordinary Differential Equations, Wiley, New York, 1962. MR 24 \# B1772.

4. H.-S. Hung, Application of Linear Spline Functions to the Numerical Solution of Volterra Integral Equations of the Second Kind, University of Wisconsin Comput. Sci. Tech. Rep. No. 27, 1968.

5. H.-S. HUNG, The Numerical Solution of Differential and Integral Equations by Spline Functions, Math. Res. Center Tech. Rep. No. 1053, Mathematics Research Center, University of Wisconsin, Madison, Wis., 1970.

6. G. A. KEMPER, "Linear multistep methods for a class of functional differential equations," Numer. Math., Band 19, 1972, pp. 361-372.

7. P. LiNZ, "Linear multistep methods for Volterra integro-differential equations," $J$. Assoc. Comput. Mach., v. 16, 1969, pp. 295-301. MR 39 \#1143. 